\title{
Existence and Hölder Regularity of the Fractional Landau-Lifshitz Equation without Gilbert Damping Term
}

\author{
Lijun Wang, ${ }^{1}$ Jingna $\mathrm{Li}^{1}{ }^{1}$ and $\mathrm{Li} \mathrm{Xia}^{2}$ \\ ${ }^{1}$ Department of Mathematics, Jinan University, Guangzhou 510632, China \\ ${ }^{2}$ Department of Mathematics, Guangdong University of Finance \& Economics, Guangzhou 510320, China \\ Correspondence should be addressed to Jingna Li; jingna8005@hotmail.com
}

Received 15 September 2013; Accepted 6 November 2013

Academic Editor: Natig M. Atakishiyev

Copyright (c) 2013 Lijun Wang et al. This is an open access article distributed under the Creative Commons Attribution License, which permits unrestricted use, distribution, and reproduction in any medium, provided the original work is properly cited.

Existence and Hölder regularity of weak solutions to the fractional Landau-Lifshitz equation without Gilbert damping term is proved through viscosity approximation. Since the nonlinear term is nonlocal and of full order of the equation, a commutator is constructed to get the convergence of the approximating solutions.

\section{Introduction}

We study the fractional Landau-Lifshitz equation

$$
\frac{\partial m}{\partial t}=-\lambda_{1} m \times \Lambda^{2 \alpha} m+\lambda_{2} m \times\left(m \times \Lambda^{2 \alpha} m\right)
$$

where $m(x, t)$ is a three-dimensional vector representing the magnetization and $\alpha, \lambda_{1}, \lambda_{2} \geq 0$ are real numbers. $\Lambda=$ $(-\Delta)^{1 / 2}$ is the square root of the Laplacian and the so-called Zygmund operator and $\times$ denotes the cross product of $\mathbb{R}^{3}$ valued vectors. The first term $m \times \Lambda^{2 \alpha} m$ is the gyromagnetic term and the second term $m \times\left(m \times \Lambda^{2 \alpha} m\right)$ is called the Gilbert damping term. The fractional diffusion operator $\Lambda^{2 \alpha}$ is nonlocal except $\alpha=0,1,2,3, \ldots$, which means that $\Lambda^{2 \alpha} u(x)$ depends not only on $u(y)$ for $y$ near $x$ but also on $u(y)$ for all $y$.

Equation (1) plays a fundamental role in the understanding of nonequilibrium magnetism, which is an interesting problem from both scientific and technological points of view. Besides their traditional applications in the magnetic recording industry, these films are also currently being explored as alternatives to semiconductors as magnetic memory devices (MRAMs), which has given greater incentive to study this subject. Since defects, impurities, and thermal noise play important roles in the dynamics of the magnetization field in nanometer thick films, they also make an ideal playground for studying some of the nanoscale physics branches [1-4].

Fractional differential equations, which appear in several branches of physics such as viscoelasticity, electrochemistry, control, porous media, and electromagnetic, now attract the interests of many mathematicians; see, for example, $[5,6]$. A good case in point is the quasi-geostrophic equation with fractional dissipation, which has been extensively studied in the last decade see [7-9]. The fractional LandauLifshitz equation shares some similar difficulties with quasigeostrophic equation; however, the equation studied here is much more complicated in several ways. The derivative in the nonlinear convective term is local in the quasi-geostrophic equation and the fluid velocity is divergence free, but here for $\lambda_{2}=0,(1)$ is degenerate and even worse the derivative in the nonlinear term is nonlocal and of the same order as the equation, which brings new difficulties in the convergence of the approximate solutions. Hence subtle techniques must be used to overcome the difficulties.

Let us recall some previous results of the equation. When $\alpha=1$, (1) becomes the standard Landau-Lifshitz equation introduced first by Landau and Lifshitz in [10], which was widely studied in [11-16]. For general $\alpha \in(0,1]$, the interested reader can refer to [17] for mathematical theory. When $\lambda_{2}=$ 0 , (1) corresponds to Schrödinger flow which represents the conservation of angular momentum [18-21]. Numerical treatments can be found in $[22,23]$. 
In this paper, we will study local existence of weak solutions in the spatial domain $(0,2 \pi)$ with $\lambda_{2}=0$ and $\alpha \epsilon$ $[1 / 2,1]$. The main difficulty, as in many partial differential equations, is the convergence of the nonlinear terms. In our situation, we even face the problem of nonlocal differential operators, degeneracy, and nonlocal nonlinear term. For these reasons, the structure of (1) must be explored in detail.

Without loss of generality, we assume that $\lambda_{1}=1$. Actually, (1) can be written as

$$
\frac{\partial m}{\partial t}=B(m) \Lambda^{2 \alpha} m
$$

in which

$$
m=\left(\begin{array}{l}
m_{1} \\
m_{2} \\
m_{3}
\end{array}\right), \quad B(m)=\left(\begin{array}{ccc}
0 & m_{3} & -m_{2} \\
-m_{3} & 0 & m_{1} \\
m_{2} & -m_{1} & 0
\end{array}\right),
$$

with initial condition

$$
m(x, 0)=m_{0}(x)
$$

and the periodic boundary condition

$$
m(x, t)=m(x+2 \pi, t) .
$$

It is straightforward to check the following conclusions.

(1) The matrix $B(m)$ is "zero definite"; namely,

$$
\xi^{\tau} B(m) \xi=0, \quad \forall \xi, m \in \mathbb{R}^{3} .
$$

(2) The matrix $B(m)$ is singular; that is,

$$
\operatorname{det} B(m)=0, \quad \forall m \in \mathbb{R}^{3} .
$$

Hence (2) is quite different from usual quasilinear parabolic equations for the above reasons.

To approximate (2), we consider the following mollified equation:

$$
\frac{\partial m}{\partial t}=-\varepsilon \Lambda^{2 \alpha} m-m \times \Lambda^{2 \alpha} m
$$

which can be written as

$$
\frac{\partial m}{\partial t}+(\varepsilon E-B(m)) \Lambda^{2 \alpha} m=0 .
$$

The rest of this paper is divided into three parts: first, we consider the corresponding linear equation and get the regularity as a preparation to deal with (9); second, positivedefinition and uniform ellipticity of matrix $\varepsilon E-B(m)$ and choice of norm space $L^{\infty}$ ensure that Leray-Schauder fixedpoint theorem can be applied to prove the existence of weak solution to (9), and the necessary a priori estimates in order to guarantee convergence are obtained; finally, existence and Hölder regularity of weak solution to (2) is proved by taking the limit of the solution to (9), in which a commutator is constructed to get the convergence.

\section{Cauchy Problem for the Corresponding Linear Equation}

Our starting point is the linear equation

$$
\begin{aligned}
\frac{\partial m}{\partial t}+A(x, t) \Lambda^{2 \alpha} m & =f(x, t), \quad \text { in } \mathbb{T}^{n} \times(0, T), \\
m(x, 0) & =m_{0}(x), \quad \text { on } \mathbb{T}^{n},
\end{aligned}
$$

where $\mathbb{T}^{n}=\mathbb{R}^{n} / Z^{n}$ is the flat torus and $m(x, t)$ and $m_{0}(x)$ are $\mathrm{N}$-dimensional vector-valued functions. We have the following theorem about existence of solution to (10)-(11).

Theorem 1. Suppose that $N \times N$ matrix $A(x, t)$ defined on $\mathbb{T}^{n} \times$ $(0, T)$ is measurable, bounded, and uniformly elliptic; namely, there exists a constant $K$ such that

$$
A(x, t) \eta \cdot \eta \geq K|\eta|^{2}
$$

for all $N$-dimensional vectors, $f(x, t) \in L^{2}\left(0, T ; L^{2}\left(\mathbb{T}^{n}\right)\right)$, and $m_{0}(x) \in H^{\alpha}\left(\mathbb{T}^{n}\right)$. Then there exists a unique vector-valued solution to (10)-(11) such that

$$
\begin{gathered}
m(x, t) \in L^{\infty}\left(0, T ; H^{\alpha}\left(\mathbb{T}^{n}\right)\right) \bigcap L^{2}\left(0, T ; H^{2 \alpha}\left(\mathbb{T}^{n}\right)\right), \\
\frac{\partial m}{\partial t} \in L^{2}\left(0, T ; L^{2}\left(\mathbb{T}^{n}\right)\right) .
\end{gathered}
$$

Proof of Theorem 1. We apply the Galerkin method: let $\left\{\varphi_{j}\right\}$ be an orthogonal basis of $L^{2}\left(\mathbb{T}^{n}\right)$ consisting of all the eigenfunctions for the operator

$$
\begin{gathered}
\Lambda^{2 \alpha} \varphi_{j}=\lambda_{j} \varphi_{j}, \\
\varphi_{j}(0)=\varphi_{j}(2 \pi) .
\end{gathered}
$$

We are looking for approximate solutions $m_{n}(x, t)$ to (10)-(11) under the form

$$
m_{n}(x, t)=\sum_{j=1}^{n} g_{j}(t) \varphi_{j}(x),
$$

where $g_{j}$ are vector-valued functions, such that, for $1 \leqslant i \leqslant n$, there holds

$$
\begin{gathered}
\int_{\Omega}\left[\frac{\partial m_{n}}{\partial t}+A(x, t) \Lambda^{2 \alpha} m_{n}-f(x, t)\right] \varphi_{i} d x=0, \\
\int_{\Omega}\left[m_{n}(x, 0)-m_{0}(x)\right] \varphi_{i} d x=0 .
\end{gathered}
$$

These relations produce an ordinary differential system that can be writeen as

$$
\frac{\partial g}{\partial t}=F(g), \quad g(0)=g_{0}
$$

where $g=\left(g_{1}, g_{2}, \ldots, g_{n}\right)$ and $g_{0}$ is the projection of $m_{0}$ on $\left(\varphi_{1}, \varphi_{2}, \ldots \varphi_{n}\right)$. The existence of a local solution to system (18) is a classical matter. We now proceed to estimate 
the approximate solution $m_{n}$. Multiplying equality (16) by $g_{i}$ and summing for $1 \leq i \leq n$, we have

$$
\begin{aligned}
\frac{1}{2}\left\|m_{n}(\cdot, t)\right\|_{L^{2}\left(\mathbb{T}^{n}\right)}^{2} & \\
= & \int_{0}^{T} \int_{\mathbb{T}^{n}}\left(f \cdot m_{n}-A(x, t) \Lambda^{2 \alpha} m_{n} \cdot m_{n}\right) d x d t \\
& +\frac{1}{2}\left\|m_{0}\right\|_{L^{2}\left(\mathbb{T}^{n}\right)}^{2} .
\end{aligned}
$$

Multiplying equality (16) by $\lambda_{i} g_{i}$ and summing for $1 \leq i \leq n$, we have

$$
\begin{gathered}
\frac{1}{2}\left\|\Lambda^{\alpha} m_{n}(\cdot, t)\right\|_{L^{2}\left(\mathbb{T}^{n}\right)}^{2}+\int_{0}^{T} \int_{\mathbb{T}^{n}} A(x, t) \Lambda^{2 \alpha} m_{n} \cdot \Lambda^{2 \alpha} m_{n} d x d t \\
=\int_{0}^{T} \int_{\mathbb{T}^{n}} f \cdot \Lambda^{2 \alpha} m_{n} d x d t+\frac{1}{2}\left\|\Lambda^{\alpha} m_{0}\right\|_{L^{2}\left(\mathbb{T}^{n}\right)}^{2} .
\end{gathered}
$$

Adding (19) to (20), we get

$$
\begin{aligned}
& \frac{1}{2}\left\|m_{n}(\cdot, t)\right\|_{L^{2}\left(\mathbb{T}^{n}\right)}^{2}+\frac{1}{2}\left\|\Lambda^{\alpha} m_{n}(\cdot, t)\right\|_{L^{2}\left(\mathbb{T}^{n}\right)}^{2} \\
& \quad+\int_{0}^{T} \int_{\mathbb{T}^{n}} A(x, t) \Lambda^{2 \alpha} m_{n} \cdot \Lambda^{2 \alpha} m_{n} d x d t \\
& =\frac{1}{2}\left(\left\|m_{0}\right\|_{L^{2}\left(\mathbb{T}^{n}\right)}^{2}+\left\|\Lambda^{\alpha} m_{0}\right\|_{L^{2}\left(\mathbb{T}^{n}\right)}^{2}\right) \\
& \quad+\int_{0}^{T} \int_{\mathbb{T}^{n}}\left(f \cdot m_{n}+f \cdot \Lambda^{2 \alpha} m_{n}\right) d x d t \\
& \quad-\int_{0}^{T} \int_{\mathbb{T}^{n}} A(x, t) \Lambda^{2 \alpha} m_{n} \cdot m_{n} d x d t \\
& \leq \frac{1}{2}\left(\left\|m_{0}\right\|_{L^{2}\left(\mathbb{T}^{n}\right)}^{2}+\left\|\Lambda^{\alpha} m_{0}\right\|_{L^{2}\left(\mathbb{T}^{n}\right)}^{2}\right)+C\|f\|_{L^{2}\left(\mathbb{T}^{n} \times(0, T)\right)}^{2} \\
& \quad+C \int_{0}^{T} \int_{\mathbb{T}^{n}}\left|m_{n}\right|^{2} d x d t+\frac{K}{2}\left\|\Lambda^{2 \alpha} m_{n}\right\|_{L^{2}\left(\mathbb{T}^{n} \times(0, T)\right)}^{2} .
\end{aligned}
$$

Since $\quad \int_{0}^{T} \int_{\mathbb{T}^{n}} A(x, t) \Lambda^{2 \alpha} m_{n} \quad \cdot \quad \Lambda^{2 \alpha} m_{n} d x d t$ $K\left\|\Lambda^{2 \alpha} m_{n}\right\|_{L^{2}\left(\mathbb{T}^{n} \times(0, T)\right)}^{2}$, by Gronwall's inequality, we have

$$
\begin{gathered}
\sup _{0 \leq t \leq T}\left\|m_{n}(\cdot, t)\right\|_{H^{\alpha}\left(\mathbb{T}^{n}\right)}^{2}+\left\|m_{n}\right\|_{L^{2}\left(\mathbb{T}^{n} \times(0, T)\right)}^{2} \\
\leq C\left(\left\|m_{0}\right\|_{H^{\alpha}\left(\mathbb{T}^{n}\right)}^{2}+\|f\|_{L^{2}\left(\mathbb{T}^{n} \times(0, T)\right)}^{2}\right), \\
\left\|\Lambda^{2 \alpha} m_{n}\right\|_{L^{2}\left(\mathbb{T}^{n} \times(0, T)\right)}^{2} \leq C\left(\left\|m_{0}\right\|_{H^{\alpha}\left(\mathbb{T}^{n}\right)}^{2}+\|f\|_{L^{2}\left(\mathbb{T}^{n} \times(0, T)\right)}^{2}\right) .
\end{gathered}
$$

Taking the inner product of $\partial m_{n} / \partial t$ and (10) and integrating over $\mathbb{T}^{n} \times(0, T)$, we have

$$
\begin{aligned}
\int_{0}^{T} \int_{\mathbb{T}^{n}}\left|\frac{\partial m_{n}}{\partial t}\right|^{2} d x d t \\
=\int_{0}^{T} \int_{\mathbb{T}^{n}}\left(f-A(x, t) \Lambda^{2 \alpha} m_{n}\right) \cdot \frac{\partial m_{n}}{\partial t} d x d t \\
\leq C \int_{0}^{T} \int_{\mathbb{T}^{n}}|f|^{2} d x d t+C \int_{0}^{T} \int_{\mathbb{T}^{n}}\left|\Lambda^{2 \alpha} m_{n}\right|^{2} d x d t \\
\quad+\frac{1}{2} \int_{0}^{T} \int_{\mathbb{T}^{n}}\left|\frac{\partial m_{n}}{\partial t}\right|^{2} d x d t .
\end{aligned}
$$

Hence

$$
\left\|\frac{\partial m_{n}}{\partial t}\right\|_{L^{2}\left(\mathbb{T}^{n} \times(0, T)\right)}^{2} \leq C\left(\left\|m_{0}\right\|_{H^{\alpha}\left(\mathbb{T}^{n}\right)}^{2}+\|f\|_{L^{2}\left(\mathbb{T}^{n} \times(0, T)\right)}^{2}\right) .
$$

Actually, if the matrix $A(x, t)$ is retrained to a small class of good function matrix, one can get higher regularity of solution to (10)-(11).

Since the right-hand member of equality (22) and (24) is uniformly bounded, thus the solution $g$ can be extended to all time, and we can extract from $m_{n}$ a subsequence (still denoted by $m_{n}$ ) such that

$$
\begin{aligned}
& m_{n} \rightarrow m s w, \quad \text { in } L^{2}\left(0, T ; H^{2 \alpha}\left(\mathbb{T}^{n}\right)\right) \text { weakly* } \\
& \frac{\partial m_{n}}{\partial t} \rightarrow \frac{\partial m}{\partial t}, \quad \text { in } L^{2}\left(0, T ; L^{2}\left(\mathbb{T}^{n}\right)\right) \text { weakly. }
\end{aligned}
$$

Hence, we know that [24]

$$
\begin{array}{r}
m_{n} \longrightarrow m, \quad \text { strongly in } L^{2}\left(0, T ; L^{2}\left(\mathbb{T}^{n}\right)\right) \\
\text { a.e. in } \mathbb{T}^{n} \times[0, T] .
\end{array}
$$

Passing to the limit $(n \rightarrow \infty)$, we find a weak solution to (10)-(11). From (16), and taking the limit $n \rightarrow \infty$, we deduce that, for all $\varphi$ in vectors $\left(\varphi_{1}, \varphi_{2}, \ldots, \varphi_{n}\right)$, there holds

$$
\int_{\mathbb{T}^{n}} \frac{\partial m_{n}}{\partial t} \varphi+\int_{\mathbb{T}^{n}} A(x, t) \Lambda^{2 \alpha} m_{n} \varphi-\int_{\mathbb{T}^{n}} f(x, t) \varphi d x=0 .
$$

By a density argument, we also obtain formula (27) for all $\varphi$ in $H^{\alpha}\left(Q_{T}\right) Q_{T}=\mathbb{T}^{n} \times[0, T]$.

Theorem 2. Suppose that:

(i) $N \times N$ matrix $A(x, t)$ defined on $\mathbb{T}^{n} \times(0, T)$ is measurable, bounded, and uniformly elliptic; namely, there exists a constant $K$ such that

$$
A(x, t) \eta \cdot \eta \geq K|\eta|^{2}
$$

for all $N$-dimensional vectors $\eta, \Delta A(x, t)$ is bounded, and $|\nabla A(x, t)|_{\infty} \leq K / \sqrt{5} \delta$, where $\delta$ is the Sobolev embedding constant satisfying

$$
\|\nabla f\|_{L^{2}\left(\mathbb{T}^{n} \times(0, T)\right)} \leq \delta\|\Delta f\|_{L^{2}\left(\mathbb{T}^{n} \times(0, T)\right)} ;
$$


(ii) $f(x, t) \in L^{2}\left(0, T ; H^{2}\left(\mathbb{T}^{n}\right)\right), m_{0}(x) \in H^{\alpha+2}\left(\mathbb{T}^{n}\right)$. Then there exists a unique vector-valued solution to (10)-(11) such that

$$
\begin{aligned}
& m(x, t) \in L^{\infty}\left(0, T ; H^{\alpha+2}\left(\mathbb{T}^{n}\right)\right) \bigcap H^{1}\left(\mathbb{T}^{n} \times(0, T)\right), \\
& \sup _{0 \leq t \leq T}\|m(\cdot, t)\|_{H^{\alpha+2}\left(\mathbb{T}^{n}\right)}^{2}+\left\|\frac{\partial m}{\partial t}\right\|_{L^{2}\left(\mathbb{T}^{n} \times(0, T)\right)}^{2}+\|m\|_{L^{2}\left(0, T ; H^{2 \alpha+2}\left(\mathbb{T}^{n}\right)\right)}^{2} \\
& \quad \leq C\left(\left\|m_{0}\right\|_{H^{\alpha+2}\left(\mathbb{T}^{n}\right)}^{2}+\|f\|_{L^{2}\left(0, T ; H^{2}\left(\mathbb{T}^{n}\right)\right)}^{2}\right),
\end{aligned}
$$

in which constant $C$ is dependent $A(x, t)$ independent of $m(x, t)$.

Proof. Let the operator $\Lambda^{2}$ act on (10); we have

$$
\begin{gathered}
-\Delta m_{t}-\Delta A(x, t) \Lambda^{2 \alpha} m+A(x, t) \Lambda^{2 \alpha+2} m \\
-\nabla A(x, t) \cdot \nabla \Lambda^{2 \alpha} m=-\Delta f(x, t) .
\end{gathered}
$$

Taking the inner product of $\Lambda^{2 \alpha+2} m$ and (31) and integrating over $\mathbb{T}^{n} \times(0, T)$, we have

$$
\begin{aligned}
\frac{1}{2} \int_{\mathbb{T}^{n}} & \left|\Lambda^{\alpha+2} m\right|^{2} d x+\int_{0}^{T} \int_{\mathbb{T}^{n}} A(x, t) \Lambda^{2 \alpha+2} m \cdot \Lambda^{2 \alpha+2} m d x d t \\
= & -\int_{0}^{T} \int_{\mathbb{T}^{n}} \Delta f \cdot \Lambda^{2 \alpha+2} m d x d t \\
& +\int_{0}^{T} \int_{\mathbb{T}^{n}} \Delta A(x, t) \Lambda^{2 \alpha} m \cdot \Lambda^{2 \alpha+2} m d x d t \\
& +\int_{0}^{T} \int_{\mathbb{T}^{n}} \nabla A(x, t) \nabla \Lambda^{2 \alpha} m \cdot \Lambda^{2 \alpha+2} m d x d t \\
& +\frac{1}{2} \int_{\mathbb{T}^{n}}\left|\Lambda^{\alpha+2} m_{0}\right|^{2} d x \\
\leq & \frac{1}{2}\left\|\Lambda^{\alpha+2} m_{0}\right\|_{L^{2}\left(\mathbb{T}^{n}\right)}^{2}+\frac{1}{K}\|\Delta f\|_{L^{2}\left(\mathbb{T}^{n} \times(0, T)\right)}^{2} \\
& +\frac{K}{4}\left\|\Lambda^{2 \alpha+2} m\right\|_{L^{2}\left(\mathbb{T}^{n} \times(0, T)\right)}^{2} \\
& +\frac{1}{K}\left\|\Delta A(x, t) \Lambda^{2 \alpha} m\right\|_{L^{2}\left(\mathbb{T}^{n} \times(0, T)\right)}^{2} \\
& +\frac{K}{4}\left\|\Lambda^{2 \alpha+2} m\right\|_{L^{2}\left(\mathbb{T}^{n} \times(0, T)\right)}^{2 \alpha+2} m \|_{L^{2}\left(\mathbb{T}^{n} \times(0, T)\right)}^{2} \\
& +\frac{1}{K}\left\|\nabla A(x, t) \nabla \Lambda^{2 \alpha} m\right\|_{L^{2}\left(\mathbb{T}^{n} \times(0, T)\right)}^{2} \\
&
\end{aligned}
$$

Note that

$$
\begin{gathered}
|\nabla A(x, t)|_{\infty}<\frac{K}{\sqrt{5} \delta}, \\
\left\|\nabla \Lambda^{2 \alpha} m\right\|_{L^{2}\left(\mathbb{T}^{n} \times(0, T)\right)} \leq \delta\left\|\Lambda^{2 \alpha+2} m\right\|_{L^{2}\left(\mathbb{T}^{n} \times(0, T)\right)} \text {, and } \\
\Delta A(x, t) \text { is bounded. }
\end{gathered}
$$

Reporting (33) in (32) and taking into account

$$
\begin{aligned}
& \int_{0}^{T} \int_{\mathbb{T}^{n}} A(x, t) \Lambda^{2 \alpha+2} m \cdot \Lambda^{2 \alpha+2} m d x d t \\
& \quad \geq K\left\|\Lambda^{2 \alpha_{+} 2} m\right\|_{L^{2}\left(\mathbb{T}^{n} \times(0, T)\right)}^{2},
\end{aligned}
$$

we have

$$
\begin{aligned}
& \sup _{0 \leq t \leq T}\left\|\Lambda^{\alpha+2} m(\cdot, t)\right\|_{L^{2}\left(\mathbb{T}^{n}\right)}^{2}+\left\|\Lambda^{2 \alpha+2} m\right\|_{L^{2}\left(\mathbb{T}^{n} \times(0, T)\right)}^{2} \\
& \quad \leq C\left(\left\|m_{0}\right\|_{H^{\alpha+2}\left(\mathbb{T}^{n}\right)}^{2}+\|f\|_{L^{2}\left(0, T: H^{2}\left(\mathbb{T}^{n}\right)\right)}^{2}\right) .
\end{aligned}
$$

\section{Cauchy Problem for the Mollified Equation}

To get existence of weak solution to (2), we consider the following approximate equation:

$$
\frac{\partial m}{\partial t}=-\varepsilon \Lambda^{2 \alpha} m-m \times \Lambda^{2 \alpha} m
$$

which is called mollified equation. In this section and next section, we assume that the spatial variable $x \in(0,2 \pi)$. By Leray-Schauder fixed-point theorem, we have the following theorem.

Theorem 3. Suppose that $m_{0}(x) \in H^{\alpha}(0,2 \pi)$, then there exists a unique weak solution to (36) with initial-boundary condition (4) and (5) such that

$$
m(x, t) \in L^{\infty}\left(0, T ; H^{\alpha}(0,2 \pi)\right)
$$

where $Q_{T}=(0,2 \pi) \times(0, T)$.

Proof. First, the mapping $T_{\lambda}: L^{\infty}\left(Q_{T}\right) \rightarrow L^{\infty}\left(Q_{T}\right)$ is defined as follows. For each $u \in L^{\infty}\left(Q_{T}\right), m=T_{\lambda}(u)$ is a solution to

$$
\frac{\partial m}{\partial t}=-\varepsilon \Lambda^{2 \alpha} m-\lambda u \times \Lambda^{2 \alpha} m
$$

with initial condition (4), in which $0 \leq \lambda \leq 1$. By Theorem 1 , we know that $m=T_{\lambda}(u)$ is the unique solution to (38) with initial-boundary condition (4) and (5); moreover, $m \in$ $L^{\infty}\left(0, T ; H^{1}(0,2 \pi)\right)$.

Obviously, for all $\lambda$, the mapping $T_{\lambda}$ is continuous; and for any bounded closed set of $L^{\infty}\left(Q_{T}\right), T_{\lambda}$ is uniformly continuous with respect to $0 \leq \lambda \leq 1$.

To apply Leray-Schauder fixed-point theorem, we make a priori estimate on all fixed points of $T_{\lambda}$. 
Taking the inner product of $m(x, t)$ and equation

$$
\frac{\partial m}{\partial t}=-\varepsilon \Lambda^{2 \alpha} m-\lambda m \times \Lambda^{2 \alpha} m,
$$

we have

$$
m \cdot \frac{\partial m}{\partial t}=-\varepsilon m \cdot \Lambda^{2 \alpha} m-\lambda\left(m \times \Lambda^{2 \alpha} m\right) \cdot m .
$$

Integrating (40) over $Q_{\tau}(0 \leq \tau \leq T)$, we get

$$
\|m(\cdot, \tau)\|_{L^{2}(0,2 \pi)}^{2}+2 \varepsilon\left\|\Lambda^{\alpha} m\right\|_{L^{2}\left(Q_{\tau}\right)}^{2} \leq\left\|m_{0}\right\|_{L^{2}(0,2 \pi)}^{2},
$$

in which $0 \leq \lambda \leq 1,0 \leq \tau \leq T$. Hence

$$
\sup _{0 \leq t \leq T}\|m(\cdot, t)\|_{L^{2}(0,2 \pi)} \leq C_{1}
$$

in which $C_{1}$ is a constant independent of $\varepsilon, \lambda$.

Taking the inner product of $\Lambda^{2 \alpha} m(x, t)$ and (39), we obtain

$$
\Lambda^{2 \alpha} m \cdot \frac{\partial m}{\partial t}=-\varepsilon \Lambda^{2 \alpha} m \cdot \Lambda^{2 \alpha} m-\lambda \Lambda^{2 \alpha} m \cdot\left(m \times \Lambda^{2 \alpha} m\right) .
$$

Integrating $(43)$ over $(0,2 \pi)$ with respect to variable $x$ leads to

$$
\frac{1}{2} \frac{d}{d t}\left\|\Lambda^{\alpha} m(\cdot, t)\right\|_{L^{2}(0,2 \pi)}^{2}+\varepsilon \int_{0}^{2 \pi}\left|\Lambda^{2 \alpha} m\right|^{2} d x \leq 0 .
$$

Obviously,

$$
\sup _{0 \leq t \leq T}\left\|\Lambda^{\alpha} m(\cdot, t)\right\|_{L^{2}(0,2 \pi)} \leq C_{2}
$$

in which $C_{2}$ is a constant independent of $\lambda$, $\varepsilon$. From (42), for each $\varepsilon>0$, we have

$$
\|m\|_{L^{2}\left(0, T ; H^{2 \alpha}(0,2 \pi)\right)} \leq C .
$$

In view of (42), (45), and (46), Sobolev embedding theorem gives the desired result.

For small initial data $m_{0}(x)$, we can get higher regularity of the solution to (36).

Theorem 4. Suppose that $m_{0}(x) \in H^{\alpha+2}(0,2 \pi), \alpha \in(1 / 2,1]$, and $\left\|m_{0}(x)\right\|_{H^{\alpha+2}}^{2} \leq 1 / M T$, where $M=o(1 / \varepsilon)$ is a certain constant, then there exists a unique weak solution to (36) with initial-boundary condition (4) and (5), such that

$$
m(x, t) \in L^{\infty}\left(0, T ; H^{\alpha+2}(0,2 \pi)\right) .
$$

Proof. Let the operator $\Lambda^{2}$ act on (39) we get

$$
\begin{aligned}
-\Delta m_{t}= & -\varepsilon \Lambda^{2 \alpha+2} m+\lambda \Delta m \times \Lambda^{2 \alpha} m \\
& -\lambda m \times \Lambda^{2 \alpha+2} m+\lambda \nabla m \times \nabla \Lambda^{2 \alpha} m .
\end{aligned}
$$

Taking the inner product of $\Lambda^{2 \alpha+2} m$ and (48) and integrating over $(0,2 \pi)$, we have

$$
\begin{aligned}
\frac{1}{2} \frac{d}{d t} \int_{0}^{2 \pi}\left|\Lambda^{\alpha+2} m\right|^{2} d x+\varepsilon \int_{0}^{2 \pi}\left|\Lambda^{2 \alpha+2} m\right|^{2} d x \\
=\lambda \int_{0}^{2 \pi}\left(\Delta m \times \Lambda^{2 \alpha} m\right) \cdot \Lambda^{2 \alpha+2} m d x \\
\quad+\lambda \int_{0}^{2 \pi}\left(\nabla m \times \nabla \Lambda^{2 \alpha} m\right) \cdot \Lambda^{2 \alpha+2} m d x \\
\leq \frac{1}{\varepsilon}\left\|\Lambda^{2 \alpha} m\right\|_{L^{\infty}}^{2}\|\Delta m\|_{L^{2}}^{2} \\
\quad+\frac{1}{\varepsilon}\|\nabla m\|_{L^{\infty}}^{2}\left\|\nabla \Lambda^{2 \alpha} m\right\|_{L^{2}}^{2}+\frac{\varepsilon}{2}\left\|\Lambda^{2 \alpha+2} m\right\|_{L^{2}}^{2} \\
\leq \frac{M}{2}\left\|\Lambda^{\alpha+2} m\right\|_{L^{2}}^{4}+\frac{\varepsilon}{2}\left\|\Lambda^{2 \alpha+2} m\right\|_{L^{2}}^{2} .
\end{aligned}
$$

Note that $M=o(1 / \varepsilon)$ is a constant; hence by Lemma 5, which will be proved later, we have

$$
\sup _{0 \leq t \leq T}\left\|\Lambda^{\alpha+2} m\right\|_{L^{2}(0,2 \pi)}^{2} \leq C_{3}
$$

in which $C_{3}$ is independent of $\varepsilon, \lambda$. From (49), for each $\varepsilon>0$, we have

$$
\|m\|_{L^{2}\left(0, T ; H^{2 \alpha+2}(0,2 \pi)\right)} \leq C .
$$

Lemma 5. Let $f(t)$ be nonnegative continuous functions for $0 \leq t \leq T$. Suppose that $f(0)<1 / k T$ and

$$
f(t) \leq k \int_{0}^{t} f^{2}(\tau) d \tau+f(0), \quad 0 \leq t \leq T,
$$

where $k$ is a constant. Then

$$
f(t) \leq \frac{f(0)}{1-k T f(0)}
$$

holds for $0 \leq t \leq T$.

Proof. Define

$$
v(t)=k \int_{0}^{t} f^{2}(\tau) d \tau+f(0) .
$$

Then the function $v(t)$ is nondecreasing, $v(0)=f(0)$, and

$$
\frac{d v(t)}{d t}=k f^{2}(t) \leq k v^{2}(t)
$$

since $f(t) \leq v(t) \leq v(T)$. According to (55), the function $z(t)=-1 / v(t)$ satisfies

$$
\frac{d z(t)}{d t}=\frac{v^{\prime}(t)}{v^{2}(t)}=\frac{k f^{2}(t)}{v^{2}(t)} \leq k .
$$


Integrating (56) from 0 to $t$ yields

$$
z(t) \leq k t+z(0)
$$

or

$$
-\frac{1}{v(t)} \leq k t-\frac{1}{v(0)}
$$

that is

$$
v(t) \leq \frac{v(0)}{1-k t v(0)}
$$

Hence

$$
f(t) \leq \frac{f(0)}{1-k T f(0)}
$$

\section{Convergence Process}

Before we prove existence of weak solution to the fractional Landau-Lifshitz model without Gilbert term (2), we first recall two Lemmas in $[25,26]$, respectively.

Lemma 6. Suppose that $s>0$ and $p \in(1,+\infty)$. If $f, g \in \mathcal{S}$, the Schwartz class, then

$$
\begin{gathered}
\left\|\Lambda^{s}(f g)-f \Lambda^{s} g\right\|_{L^{p}} \\
\leq C\left(\|\nabla f\|_{L^{p_{1}}}\|g\|_{\dot{W}^{s-1, p_{2}}}+\|f\|_{\dot{W}^{s, p_{3}}}\|g\|_{L^{p_{4}}}\right), \\
\left\|\Lambda^{s}(f g)\right\|_{L^{p}} \leq C\left(\|f\|_{L^{p_{1}}}\|g\|_{\dot{W}^{s, p_{2}}}+\|f\|_{\dot{W}^{s, p_{3}}}\|g\|_{L^{p^{4}}}\right),
\end{gathered}
$$

with $p_{2}, p_{3} \in(1,+\infty)$ such that

$$
\frac{1}{p}=\frac{1}{p_{1}}+\frac{1}{p_{2}}=\frac{1}{p_{3}}+\frac{1}{p_{4}}
$$

where $\dot{W}^{k, p}$ is the homogeneous Sobolev space and the $\dot{W}^{k, p}$ norm of $f$ is defined by

$$
\|f\|_{\dot{W}^{k, p}}=\left\|\mathscr{F}^{-1}\left(|\xi|^{s} \widehat{f}(\xi)\right)\right\|_{L^{p}} .
$$

The following Lemma is due to Gagliardo and Nirenberg, see [11].

Lemma 7. Let $\Omega$ be $\mathbb{R}^{n}$ or a bounded Lipschitz domain in $\mathbb{R}^{n}$ with $\partial \Omega$, and let $u$ be any function in $W^{m, r}(\Omega) \cap L^{q}(\Omega), 1 \leq$ $r, q \leq+\infty$. For any integer $j, 0 \leq j<m$, and for any number $a$ in the interval $j / m \leq a \leq 1$, set

$$
\frac{1}{p}=\frac{j}{n}+a\left(\frac{1}{r}-\frac{m}{n}\right)+(1-a) \frac{1}{q} .
$$

If $m-j-(n / r)$ is not a nonnegative integer, then

$$
\left\|\nabla^{j} u\right\|_{L^{p}(\Omega)} \leq C\|u\|_{W^{m, r}(\Omega)}^{a}\|u\|_{L^{q}(\Omega)}^{1-a} .
$$

If $m-j-(n / r)$ is a nonnegative integer, then (65) holds for $a=j / m$. The constant $C$ depends only on $r, q, m, j, a$, and the shape of $\Omega$.

From (45), we conclude the following.
Lemma 8. Solutions to (36) satisfy

$$
\sup _{0 \leq t \leq T}\left\|m^{\varepsilon}(\cdot, t)\right\|_{H^{\alpha}(0,2 \pi)} \leq C,
$$

in which $C$ is independent of $\varepsilon$.

For the uniform bound of $\partial m^{\varepsilon} / \partial t$, we have the following lemma.

Lemma 9. $\partial m^{\varepsilon} / \partial t$ in (36) satisfies

$$
\sup _{0 \leq t \leq T}\left\|\frac{\partial m^{\varepsilon}}{\partial t}(\cdot, t)\right\|_{H^{-k}(0,2 \pi)} \leq C,
$$

in which $1 / 2<k<1$ and $C$ is independent of $\varepsilon$.

Proof. For all $\psi(x) \in\left(H^{\alpha}(0,2 \pi)\right)^{3}$, we have

$$
\begin{aligned}
\int_{-\infty}^{+\infty} & \psi \cdot \frac{\partial m^{\varepsilon}}{\partial t} d x \\
= & -\varepsilon \int_{-\infty}^{+\infty} \Lambda^{\alpha} \psi(x) \cdot \Lambda^{\alpha} m^{\varepsilon} d x \\
& -\int_{-\infty}^{+\infty}\left(m^{\varepsilon} \times \Lambda^{2 \alpha} m^{\varepsilon}\right) \cdot \psi(x) d x \\
= & -\varepsilon \int_{-\infty}^{+\infty} \Lambda^{\alpha} \psi(x) \cdot \Lambda^{\alpha} m^{\varepsilon} d x \\
& -\int_{-\infty}^{+\infty}\left(\psi(x) \times m^{\varepsilon}\right) \cdot \Lambda^{2 \alpha} m^{\varepsilon} d x \\
= & -\varepsilon \int_{-\infty}^{+\infty} \Lambda^{\alpha} \psi(x) \cdot \Lambda^{\alpha} m^{\varepsilon} d x \\
& -\int_{-\infty}^{+\infty} \Lambda^{\alpha}\left(\psi(x) \times m^{\varepsilon}\right) \cdot \Lambda^{\alpha} m^{\varepsilon} d x \\
\leq & \|\psi\|_{L^{\infty}}\left\|\Lambda^{\alpha} m^{\varepsilon}\right\|_{L^{2}}^{2}+\left\|\Lambda^{\alpha} m^{\varepsilon}\right\|_{L^{2}}\left\|\Lambda^{\alpha} \psi\right\|_{L^{4}}\left\|m^{\varepsilon}\right\|_{L^{4}} \\
\leq & C\|\psi\|_{H^{k}(0,2 \pi)}
\end{aligned}
$$

in which formula $(\vec{a} \times \vec{b}) \cdot \vec{c}=(\vec{c} \times \vec{a}) \cdot \vec{b}$; Sobolev embedding theorem and Lemma 6 are used.

The following lemma is about the uniform bound of the Hölder norm of $m^{\varepsilon}$.

Lemma 10. Solutions to (36) satisfy

$$
\left\|m^{\varepsilon}(x, t)\right\|_{C^{(2 \alpha-1) /(\alpha+1),(2 \alpha-1) /(2(\alpha+1))}\left(Q_{T}\right)} \leq C,
$$

where $C$ is independent of $\varepsilon$. 
Proof. Denote $\omega^{\varepsilon}=\int_{0}^{x} m^{\varepsilon}(\xi, t) d \xi$

$$
\begin{aligned}
& \forall \psi(x) \in H_{0}^{k}(0,2 \pi) \\
&\left|\int_{0}^{2 \pi} \psi^{\prime}(x) \omega_{t}^{\varepsilon}(x, t) d x\right|=\left|\int_{0}^{2 \pi} \psi(x) m_{t}^{\varepsilon}(x, t) d x\right| \\
& \leq C\|\psi(x)\|_{H^{k}(0,2 \pi)} \\
& \leq C\left\|\psi^{\prime}(x)\right\|_{L^{2}(0,2 \pi)}
\end{aligned}
$$

Therefore

$$
\sup _{0 \leq t \leq T}\left\|\omega_{t}^{\varepsilon}(x, t)\right\|_{L^{2}} \leq C
$$

Hence

$$
\left\{\omega^{\varepsilon}(x, t)\right\} \text { is bounded in } L^{\infty}\left(0, T ; H^{\alpha+1}(0,2 \pi)\right)
$$

$$
\bigcap W^{1, \infty}\left(0, T ; H^{1-\alpha}(0,2 \pi)\right) \text {. }
$$

Therefore, by Lemma 7,

$$
\begin{aligned}
& \left|m^{\varepsilon}\left(x, t_{2}\right)-m^{\varepsilon}\left(x, t_{1}\right)\right| \\
& =\left|\omega_{x}^{\varepsilon}\left(x, t_{2}\right)-\omega_{x}^{\varepsilon}\left(x, t_{1}\right)\right| \\
& \leq C\left\|\omega^{\varepsilon}\left(\cdot, t_{2}\right)-\omega^{\varepsilon}\left(\cdot, t_{1}\right)\right\|_{L^{2}(0,2 \pi)}^{(2 \alpha-1) / 2(\alpha+1)} \\
& \quad \times\left\|\omega^{\varepsilon}\left(\cdot, t_{2}\right)-\omega^{\varepsilon}\left(\cdot, t_{1}\right)\right\|_{H^{\alpha+1}(0,2 \pi)}^{3 / 2(\alpha+1)} \\
& \leq C\left|t_{2}-t_{1}\right|^{(2 \alpha-1) / 2(\alpha+1)} \\
& \quad \times \sup _{0 \leq t \leq T}\left\|w_{t}^{\varepsilon}(\cdot, t)\right\|_{L^{2}(0,2 \pi)}^{(2 \alpha-1) / 2(\alpha+1)} \\
& \quad \times\left\|\omega^{\varepsilon}\left(\cdot, t_{2}\right)-\omega^{\varepsilon}\left(\cdot, t_{1}\right)\right\|_{H^{\alpha+1}(0,2 \pi)}^{3 / 2(\alpha+1)} .
\end{aligned}
$$

Weak solution to (2) with initial-boundary condition (4) and (5) is defined as follows.

Definition 11. 3-dimensional vector-valued function $m(x, t)$ is called a weak solution to (2) with initial-boundary condition (4) and (5), if

$$
\begin{aligned}
& m(x, t) \in L^{\infty}\left(0, T ; H^{\alpha}(0,2 \pi)\right) \\
& \bigcap C^{(2 \alpha-1) /(\alpha+1),(2 \alpha-1) / 2(\alpha+1)}\left(Q_{T}\right), \\
& \iint_{Q_{T}}\left[\frac{\partial m}{\partial t} \cdot \varphi+\Lambda^{\alpha}(\varphi \times m) \cdot \Lambda^{\alpha} m\right] d x d t \\
& +\int_{0}^{2 \pi} \varphi(x, 0) \cdot m_{0}(x) d x=0,
\end{aligned}
$$

for all test functions $\varphi \in\left(C^{\infty}\left(Q_{T}\right)\right)^{3}$.
Theorem 12. Suppose that $m_{0}(x) \in H^{\alpha}(0,2 \pi)$; then there exists a weak solution to (2) with initial-boundary condition (4) and (5) satisfying

$$
\begin{aligned}
& m(x, t) \in L^{\infty}\left(0, T ; H^{\alpha}(0,2 \pi)\right) \\
& \bigcap C^{(2 \alpha-1) /(\alpha+1),(2 \alpha-1) / 2(\alpha+1)}\left(Q_{T}\right), \\
& \frac{\partial m}{\partial t} \in L^{\infty}\left(0, T ; H^{-\alpha}(0,2 \pi)\right) .
\end{aligned}
$$

Proof. For a solution to the mollified equation (36), we have

$$
\iint_{Q_{T}}\left[\frac{\partial m^{\varepsilon}}{\partial t} \cdot \varphi+\varepsilon \Lambda^{\alpha} \varphi \cdot \Lambda^{\alpha} m^{\varepsilon}+\Lambda^{\alpha}\left(\varphi \times m^{\varepsilon}\right) \cdot \Lambda^{\alpha} m^{\varepsilon}\right] d x d t
$$$$
+\int_{0}^{2 \pi} \varphi(x, 0) m_{0}(x) d x=0, \quad \forall \varphi \in\left(C^{\infty}\left(Q_{T}\right)\right)^{3} .
$$

By Lemmas 8 and 9,

$$
\begin{gathered}
\left\{m^{\varepsilon}(x, t)\right\} \text { is bounded in } L^{\infty}\left(0, T ; H^{\alpha}(0,2 \pi)\right) \\
\bigcap C^{(2 \alpha-1) /(\alpha+1),(2 \alpha-1) / 2(\alpha+1)}\left(Q_{T}\right), \\
\left\{\frac{\partial m^{\varepsilon}}{\partial t}(x, t)\right\} \text { is bounded in } L^{\infty}\left(0, T ; H^{-k}(0,2 \pi)\right) .
\end{gathered}
$$

Hence $m(x, t) \in C^{(2 \alpha-1) /(\alpha+1),(2 \alpha-1) / 2(\alpha+1)}\left(Q_{T}\right)$. We select a subsequence (still denoted as $\left.\left\{m^{\varepsilon}(x, t)\right\}\right)$, such that

$$
\begin{array}{r}
\left\{m^{\varepsilon}(x, t)\right\} \text { is weak }{ }^{*} \text { convengent to } m(x, t) \\
\qquad \text { in } L^{\infty}\left(0, T ; H^{\alpha}(0,2 \pi)\right),
\end{array}
$$

$\left\{m^{\varepsilon}(x, t)\right\}$ is uniformly convergent to $m(x, t)$ in $Q_{T}$, and

$$
\begin{array}{r}
\left\{\frac{\partial m^{\varepsilon}}{\partial t}(x, t)\right\} \text { is weak }{ }^{*} \text { convengent to } \frac{\partial m}{\partial t} \\
\text { in } L^{\infty}\left(0, T ; H^{-k}(0,2 \pi)\right) .
\end{array}
$$

By a theorem in [24], we have that

$$
\begin{aligned}
& \left\{m^{\varepsilon}(x, t)\right\} \text { is strongly convengent to } m(x, t) \\
& \qquad \text { in } L^{2}\left(0, T ; L^{2}(0,2 \pi)\right) .
\end{aligned}
$$

To prove that

$$
\begin{aligned}
& \iint_{Q_{T}} \Lambda^{\alpha}\left(\varphi \times m^{\varepsilon}\right) \cdot \Lambda^{\alpha} m^{\varepsilon} d x d t \\
& \quad \longrightarrow \iint_{Q_{T}} \Lambda^{\alpha}(\varphi \times m) \cdot \Lambda^{\alpha} m d x d t
\end{aligned}
$$


we have to construct a commutator. Actually,

$$
\begin{aligned}
& \iint_{\mathrm{Q}_{T}} \Lambda^{\alpha}\left(\varphi \times m^{\varepsilon}\right) \cdot \Lambda^{\alpha} m^{\varepsilon} d x d t \\
& \quad=\iint_{Q_{T}} \Lambda^{\alpha}\left(\varphi \times m^{\varepsilon}\right) \cdot \Lambda^{\alpha} m^{\varepsilon} d x d t \\
& \quad-\iint_{Q_{T}}\left(\varphi \times \Lambda^{\alpha} m^{\varepsilon}\right) \cdot \Lambda^{\alpha} m^{\varepsilon} d x d t \\
& =\iint_{Q_{T}}\left[\Lambda^{\alpha}\left(\varphi \times m^{\varepsilon}\right)-\varphi \times \Lambda^{\alpha} m^{\varepsilon}\right] \cdot \Lambda^{\alpha} m^{\varepsilon} d x d t .
\end{aligned}
$$

Similarly,

$$
\begin{aligned}
& \iint_{Q_{T}} \Lambda^{\alpha}(\varphi \times m) \cdot \Lambda^{\alpha} m d x d t \\
& \quad=\iint_{Q_{T}}\left[\Lambda^{\alpha}(\varphi \times m)-\varphi \times \Lambda^{\alpha} m\right] \cdot \Lambda^{\alpha} m d x d t .
\end{aligned}
$$

Denote $\Lambda^{\alpha}(\varphi \times u)-\varphi \times \Lambda^{\alpha} u$ by $\mathscr{L}_{\varphi}(u)$. To get (80), it suffices to prove that

$$
\begin{gathered}
\iint_{Q_{T}} \mathscr{L}_{\varphi}\left(m^{\varepsilon}\right) \Lambda^{\alpha} m^{\varepsilon}-\mathscr{L}_{\varphi}(m) \Lambda^{\alpha} m d x d t \\
=\iint_{Q_{T}}\left[\mathscr{L}_{\varphi}\left(m^{\varepsilon}\right)-\mathscr{L}_{\varphi}(m)\right] \cdot \Lambda^{\alpha} m^{\varepsilon} \\
\quad+\iint_{Q_{T}} \mathscr{L}_{\varphi}(m)\left[\Lambda^{\alpha} m^{\varepsilon}-\Lambda^{\alpha} m\right] d x d t \\
=I_{1}+I_{2} \longrightarrow 0, \quad \text { as } \varepsilon \longrightarrow 0 .
\end{gathered}
$$

Obviously $I_{2} \rightarrow 0$, as $\varepsilon \rightarrow 0$. We need to prove that $I_{1} \rightarrow 0$, as $\varepsilon \rightarrow 0$. Actually, by Lemma 6 , we can get

$$
\begin{aligned}
& \left\|\mathscr{L}_{\varphi}\left(m^{\varepsilon}\right)-\mathscr{L}_{\varphi}(m)\right\|_{L^{2}(0,2 \pi)} \\
& \quad \leq C\left(\|\nabla \varphi\|_{L^{p_{1}}}\left\|m^{\varepsilon}-m\right\|_{\dot{W}^{\alpha-1, p_{2}}}+\|\varphi\|_{\dot{W}^{\alpha, p_{3}}}\left\|m^{\varepsilon}-m\right\|_{L^{p_{4}}}\right) \\
& \quad \leq C\left(\|\nabla \varphi\|_{L^{p_{1}}}\left\|m^{\varepsilon}-m\right\|_{L^{2}}+\|\varphi\|_{\dot{W}^{\alpha, p_{3}}}\left\|m^{\varepsilon}-m\right\|_{H^{\beta}}\right) \\
& \quad \leq C\|\varphi\|_{H^{\gamma}(0,2 \pi)}\left\|m^{\varepsilon}-m\right\|_{H^{\beta}(0,2 \pi)},
\end{aligned}
$$

where $p_{2}, p_{3} \in(1,+\infty)$ are such that

$$
\begin{array}{ll}
\frac{1}{p_{1}}+\frac{1}{p_{2}}=\frac{1}{2}, & \frac{1}{p_{3}}+\frac{1}{p_{4}}=\frac{1}{2}, \\
0<\beta<\alpha<1, & \frac{p_{2}}{\alpha p_{2}-1}=2, \\
p_{4}=\frac{2}{1-2 \beta}, & 2(\gamma-1)>1 .
\end{array}
$$

Therefore $\gamma>\alpha+(1 / 2)-\left(1 / p_{3}\right)$ automatically.
By Hölder's inequality, we can show that

$$
\begin{aligned}
& \left|\iint_{Q_{T}} \Lambda^{\alpha} m^{\varepsilon} \mathscr{L}_{\varphi}\left(m^{\varepsilon}-m\right) d x d t\right| \\
& \leq C\left\|\Lambda^{\alpha} m^{\varepsilon}\right\|_{L^{2}\left(Q_{T}\right)}\|\varphi\|_{L^{2}\left(0, T ; H^{\gamma}(0,2 \pi)\right)} \\
& \quad \times\left\|m^{\varepsilon}-m\right\|_{L^{2}\left(0, T ; H^{\beta}(0,2 \pi)\right)} \longrightarrow 0, \quad \text { as } \varepsilon \longrightarrow 0 .
\end{aligned}
$$

As $\varepsilon \rightarrow 0$ in (76), we have

$$
\begin{gathered}
\iint_{Q_{T}}\left[\frac{\partial m}{\partial t} \varphi+\Lambda^{\alpha}(\varphi \times m) \cdot \Lambda^{\alpha} m\right] d x d t \\
+\int_{0}^{2 \pi} \varphi(x, 0) \cdot m_{0}(x) d x=0
\end{gathered}
$$

for all test functions $\varphi \in\left(C^{\infty}\left(Q_{T}\right)\right)^{3}$.

\section{Acknowledgment}

The paper is supported by the National Natural Science Foundation of China (no. 11201181, no. 11201311).

\section{References}

[1] A. Hubert and R. Schafer, Magnetic Domains: The Analysis of Magnetic Microstructures, Springer, Berlin, Germany, 1998.

[2] J. M. Daughton, "Magnetoresistive memory technology," Thin Solid Films, vol. 216, no. 1, pp. 162-168, 1992.

[3] B. Heinrich and J. A. C. Bland, Ultrathin Magnetic Structures I, Springer, Berlin, Germany, 1994.

[4] G. A. Prinz, “Magnetoelectronics," Science, vol. 282, no. 5394, pp. 1660-1663, 1998.

[5] K. S. Miller and B. Ross, An Introduction to the Fractional Calculus and Differential Equations, John Wiley, New Youk, NY, USA, 1993.

[6] I. Podlubny, Fractional Differential Equations, Mathematics in Science and Engeineering 198, Academic Press, San Diego, Calif, USA, 1999.

[7] P. Constantin, D. Cordoba, and J. Wu, "On the critical dissipative quasi-geostrophic equation," Indiana University Mathematics Journal, vol. 50, pp. 97-106, 2001.

[8] P. Constantin, A. J. Majda, and E. Tabak, "Formation of strong fronts in the 2-D quasigeostrophic thermal active scalar," Nonlinearity, vol. 7, no. 6, pp. 1495-1533, 1994.

[9] P. Constantin and J. Wu, "Behavior of solutions of 2D quasigeostrophic equations," SIAM Journal on Mathematical Analysis, vol. 30, no. 5, pp. 937-948, 1999.

[10] L. Landau and E. Lifshitz, "On the theory of the dispersion of magnetic permeability in ferromagnetic bodies," Ukrainian Journal of Physics, vol. 8, pp. 153-169, 1935.

[11] B. L. Guo and M. C. Hong, "The Landau-Lifshitz equation of the ferromagnetic spin chain and harmonic maps," Calculus of Variations and Partial Differential Equations, vol. 1, no. 3, pp. 311-334, 1993.

[12] M. C. Yunmei, J. D. Shijin, and L. G. Boling, "Partial regularity for two dimensional Landau-Lifshitz equations," Acta Mathematica Sinica, vol. 14, no. 3, pp. 423-432, 1998. 
[13] F. Alouges and A. Soyeur, "On global weak solutions for Landau-Lifshitz equations: existence and nonuniqueness," Nonlinear Analysis, vol. 18, no. 11, pp. 1071-1084, 1992.

[14] A. Visintin, "On Landau-Lifshitz' equations for ferromagnetism," Japan Journal of Applied Mathematics, vol. 2, no. 1, pp. 69-84, 1985.

[15] J. L. Joly, G. Métivier, and J. Rauch, "Global solutions to Maxwell equations in a ferromagnetic medium," Annales Henri Poincare, vol. 1, no. 2, pp. 307-340, 2000.

[16] G. Carbou and P. Fabrie, "Regular solutions for Landau-Lifshitz equation in a bounded domain," Differential Integral Equations, vol. 14, pp. 213-229, 2001.

[17] B. Guo and X. Pu, "The fractional Landau-Lifshitz-Gilbert equation and the heat flow of harmonic maps," Calculus of Variations and Partial Differential Equations, vol. 42, no. 1-2, pp. 1-19, 2011.

[18] P. L. Sulem, C. Sulem, and C. Bardos, "On the continuous limit for a system of classical spins," Communications in Mathematical Physics, vol. 107, no. 3, pp. 431-454, 1986.

[19] N. Chang, J. Shatah, and K. Uhlenbeck, "Schrödinger maps," Communications on Pure and Applied Mathematics, vol. 53, no. 5, pp. 590-602, 2000.

[20] J. Shatah and C. Zeng, "Schrödinger maps and antiferromagnetic chains," Communications in Mathematical Physics, vol. 262, no. 2, pp. 299-315, 2006.

[21] W. Ding, H. Tang, and C. Zeng, "Self-similar solutions of Schrödinger flows," Calculus of Variations and Partial Differential Equations, vol. 34, no. 2, pp. 267-277, 2009.

[22] N. E. Weinan and X. P. Wang, "Numerical methods for the Landau-Lifshitz equation," SIAM Journal on Numerical Analysis, vol. 38, no. 5, pp. 1647-1665, 2001.

[23] C. J. García-Cervera and X. Wang, "Spin-polarized currents in ferromagnetic multilayers," Journal of Computational Physics, vol. 224, no. 2, pp. 699-711, 2007.

[24] J. L. Lions, Quelques Methodes de Resolution des Problems Aux Limites Non Lineaire, Chinese Edition, Sun Yat-sen University Press, GuangZhou, China, 1996.

[25] R. Coifman and Y. Meyer, Nolinear Harmonic Analysis, Opertor Theory and P.D.E., Princeton University Press, Princeton, NJ, USA, 1986.

[26] A. Friedman, Partial Differential Equations, Holt, Rinehart and Winston, New York, NY, USA, 1969. 


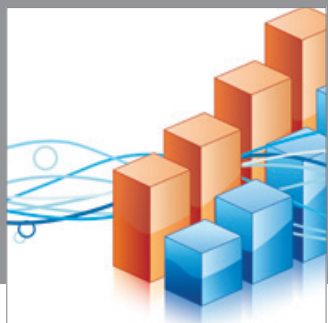

Advances in

Operations Research

mansans

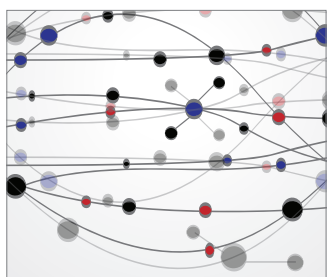

The Scientific World Journal
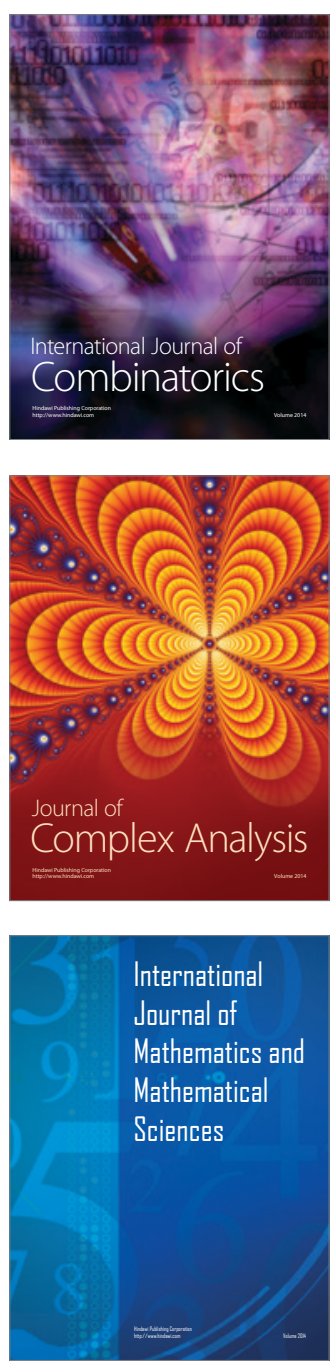
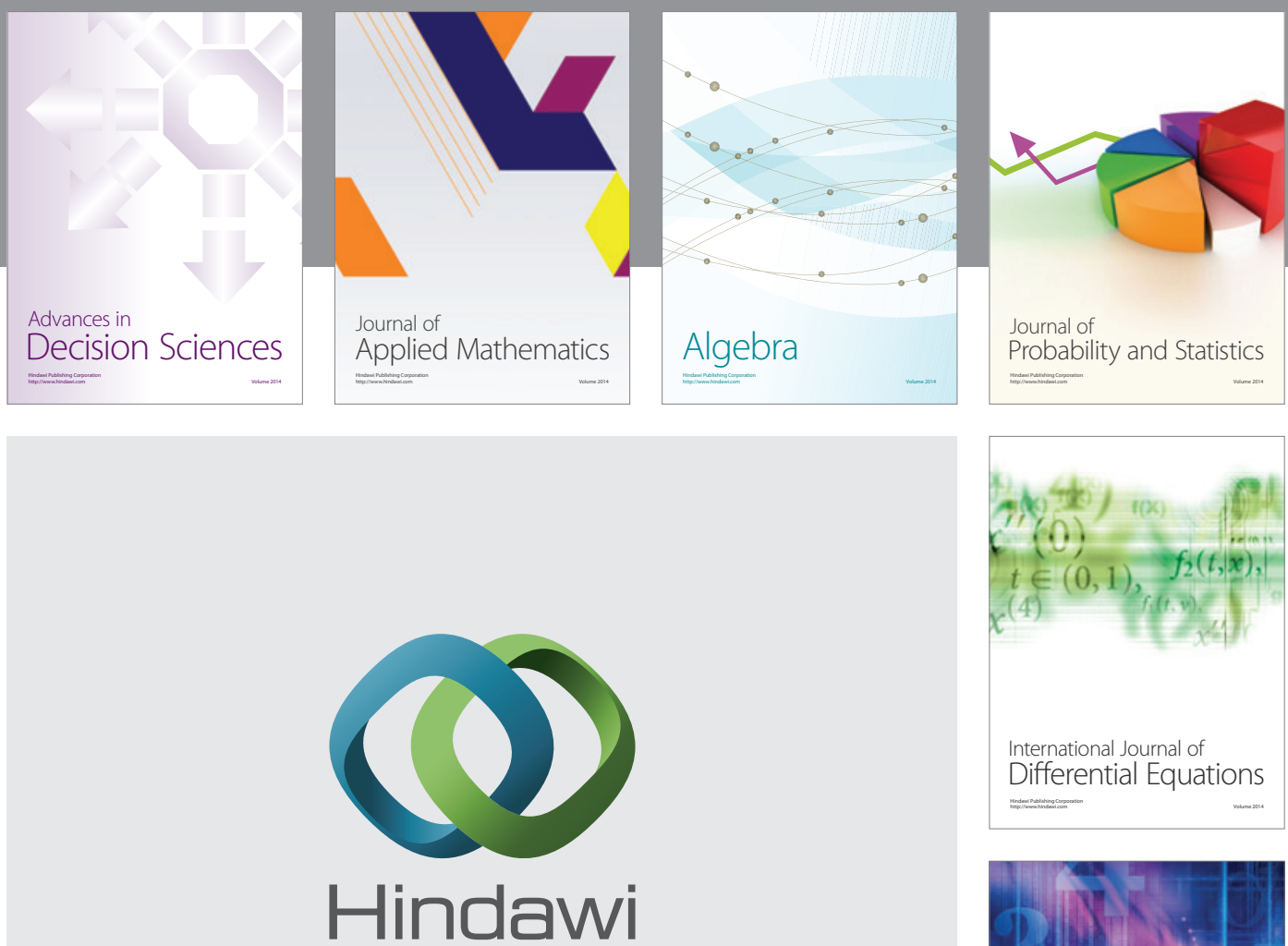

Submit your manuscripts at http://www.hindawi.com
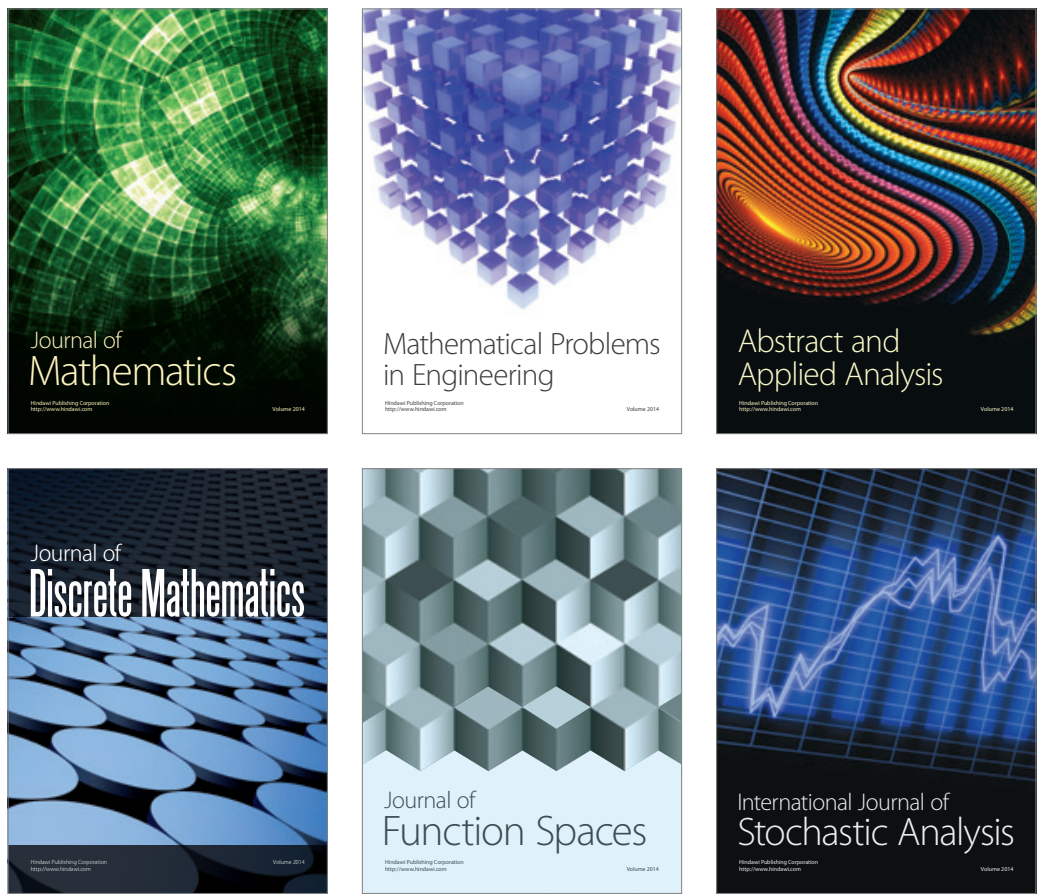

Journal of

Function Spaces

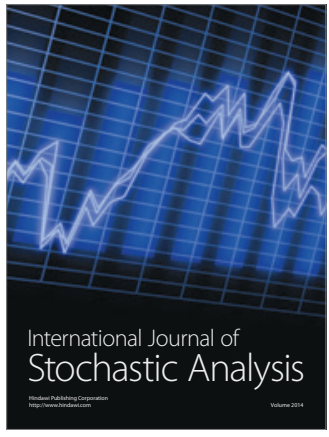

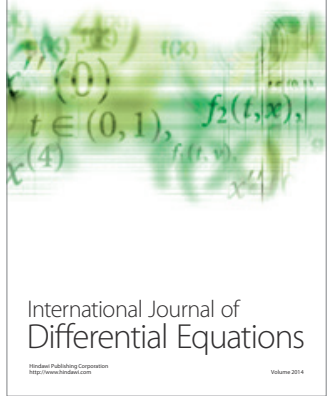
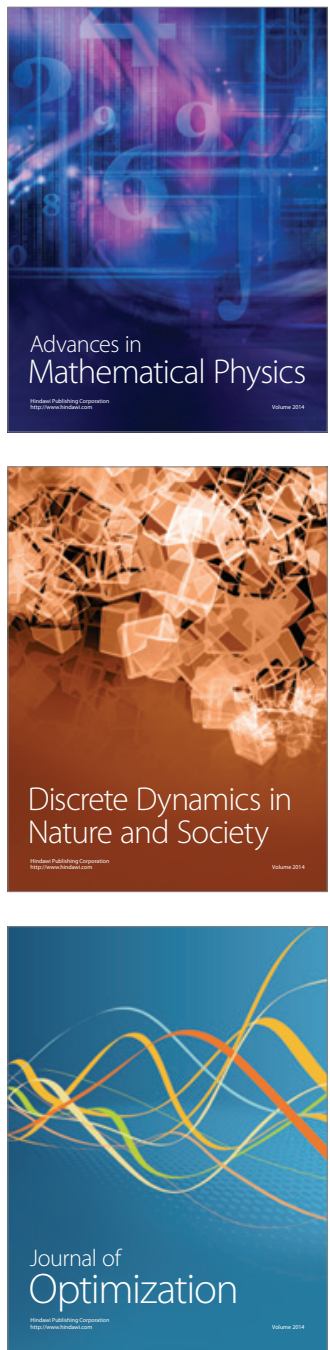